\section{A case of colonic perforation from biliary stent}

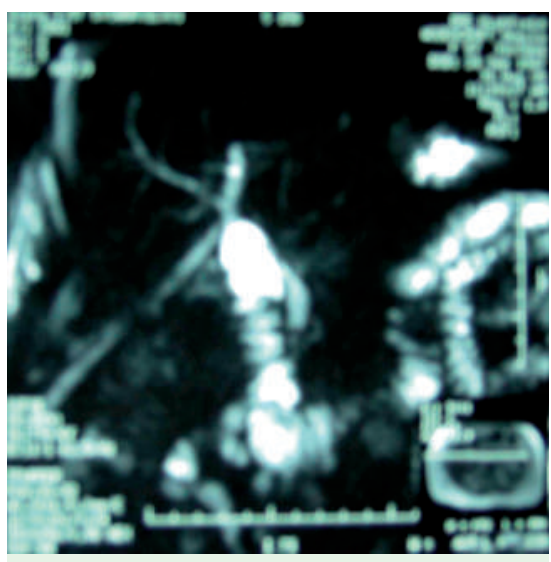

Fig. 1 Magnetic resonance cholangiopancreatography revealed a stone at the lower end of the common bile duct.

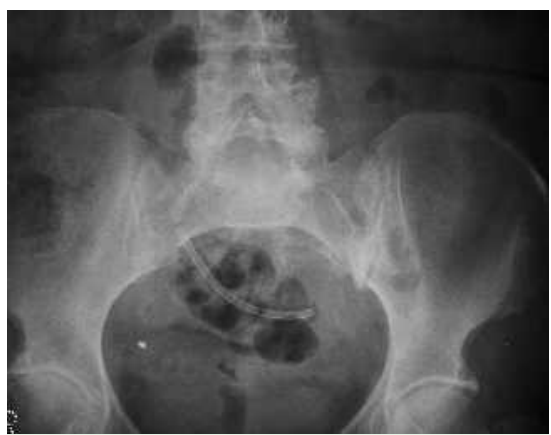

Fig. 2 Radiograph showing migrated biliary stent at pelvis.

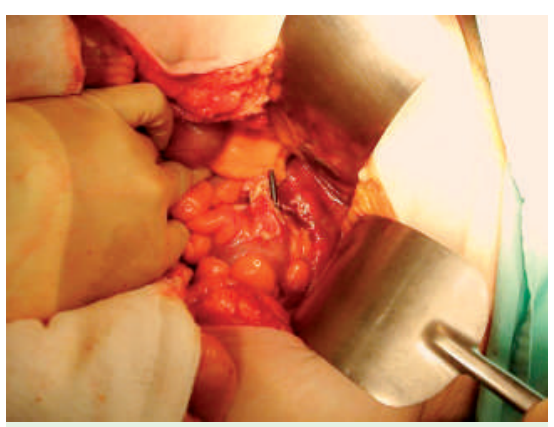

Fig. 3 Operative photograph showing perforated biliary stent through the sigmoid colon.

Plastic biliary stents are being increasingly used in benign biliary disease including postoperative bile leaks [1]. Occasionally, these stents may migrate distally, most passing spontaneously without causing problems. Perforation of the colon due to migrated stents is a very rare but reported complication.
A 57-year-old lady was referred 3 days after a laparoscopic cholecystectomy with right hypochondrial pain. Blood tests revealed leucocytosis, normal bilirubin but slightly raised alkaline phosphatase, alanine transaminase, and gamma glutamyl transferase. Ultrasonography and computed tomography (CT) scan of the abdomen demonstrated a right subphrenic fluid collection, and magnetic resonance cholangiopancreatography (MRCP) suggested a retained stone in the common bile duct ( $\bullet$ Fig. 1). A laparoscopic wash out and drainage was performed, draining $500 \mathrm{~mL}$ of bile, which ameliorated her symptoms. An endoscopic retrograde cholangiopancreatography (ERCP) demonstrated small cystic duct leak but no retained stone. So a polyethylene $10-\mathrm{Fr} 7 \mathrm{~cm}$ long biliary endoprosthesis (Cotton-Leung, Wilson-Cook Medical Inc., Winston-Salem, North Carolina, USA) was inserted. Her condition improved, bile leak stopped, abdominal drain was removed, and she was discharged from hospital.

She re-presented 4 weeks later with a 3day history of abdominal pain and no bowel movement. She was pyrexial and had peritonism in the lower abdomen. The biliary stent was seen to be present in the lower abdomen on abdominal radiograph ( $\bullet$ Fig. 2). Laparotomy revealed perforated stent through a sigmoid diverticulum with minimal contamination ( $\bullet$ Fig. 3). The stent was removed and the perforation was closed with a covering loop transverse colostomy. Her postoperative recovery was unremarkable, and 2 months later she underwent an uneventful sigmoid colectomy and closure of the colostomy.

Only nine other cases of colonic perforation (five free perforation in the peritoneum, one through the cecal diverticulum with localised abscess, and three to the adjacent organ or fistulating through the skin) have been reported [2-5]. (Not all references cited due to lack of space.) Stent perforation should be considered in a patient presenting with abdominal pain and peritonism following placement of a biliary endoprosthesis. Stents should al- ways be removed following an exchange procedure to prevent risk of perforation.

Endoscopy_UCTN_Code_CPL_1AK_2AD

K. R. Aryal, D. J. Sherlock

Hepatobilary Unit, North Manchester

General Hospital, Manchester, UK

\section{References}

1 Kaffes AJ, Hourigan L, De Luca N et al. Impact of endoscopic intervention in 100 patients with suspected postcholecystectomy bile leak. Gastrointest Endosc 2005; 61: 269275

2 Ruffolo TA, Lehmen GA, Sherman S et al. Biliary stent migration with colonic diverticular impaction. Gastrointest Endosc 1992; 38: $81-83$

3 D'Costa H, Toy E, Dennis MJS, Brown C. Intestinal perforation - an unusual complication of endoscopic biliary stenting. Br J Radiol 1994; 67: $1270-1271$

4 Schaaftsma RJH, Spoelstra P, Pakan J, Huibregtse $K$. Sigmoid perforation: a rare complication of a migrated biliary endoprosthesis. Endoscopy 1996; 28: 469-470

5 Baty V, Denis B, Bigard MA, Gaucher P. Sigmoid diverticular perforation relating to the migration of polyethylene endoprosthesis. Endoscopy 1996; 28: 781

Bibliography

DOI $10.1055 / \mathrm{s}-2007-967056$

Endoscopy 2008; 40: E54

(c) Georg Thieme Verlag KG Stuttgart · New York . ISSN 0013-726X

Corresponding author

K. R. Aryal, MS, FRCS

5 Santon Close

Wesham

Preston

PR4 3HF

UK

Fax: +44-1772-523077

rlkamal2000@hotmail.com 